\title{
ИТЕРАТИВНЫЙ АЛГОРИТМ ДИСКРЕТНОГО ОРТОГОНАЛЬНОГО ПРЕОБРАЗОВАНИЯ СИГНАЛОВ В БАЗИСЕ ДВУМЕРНЫХ ФУНКЦИЙ
}

\author{
А. А. Дудкин, А. Г. Мачнев, А. М. Селиханович \\ Государственное научное учреждение «Институт технической кибернетики НАН Беларуси», ул. Сурганова, 6. \\ 220072, г.Минск, Беларусь \\ \{doudkin,sel\}@newman.bas-net.by
}

Резюме. Предлагается паралл ельный алгоритм дискретного ортогональ-ного преобразования двумерных сигналов, который отличаетсяот известных меньшим числом операчий умножений и сложений и тем, что коэффициенты преобразования вычисляются за один раз без использования одномерных преобразо-ваний. Кроме того, алгоритм удобен для СБИС-реализаиии. На базе этого алгоритма преложены алгоритмы выделения контуров на полутоновых изображениях.

Ключевые слова: Ортогональное преобразование, 2D функиял, вычисление спектральных коэффициентов, быстрый алгоритм, преобразование Уолиа / Хаара, полутоновое изображение, выделение контуров.

\section{1. ВВЕДЕНИЕ}

Двумерные преобразования Фурье, Уолша, Хаара и другие ортогональные преобразования широко используются в обработке сигналов и цифровых изображений. Как правило, двумерные преобразования реализуются на СБИС в виде матричных систолических процессоров, при этом умножения матриц выполняется дважды [1].

Другой подход к реализации двумерных преобразований базируется на применении двумерных дискретных ортогональных функций. Многомерные функции (2D функции) Уолша и Хаара в различных системах координат представлены в [2]. 2D функции Виленкина Крестенсона, алгоритмы построения которых предложены в [3], включают в себя как функции Уолша, так и дискретные экспоненциальные функции (ДЭФ), которые используются в Фурье преобразовании.

В статье описывается новый алгоритм дискретного ортогонального преобразования двумерных сигналов, который имеет два основных отличия от известных алгоритмов. Во-первых, он имеет меньшее число операций умножений и сложений. Во-вторых, коэффициенты преобразования вычисляются за один раз без вычисления одномерных преобразований. Кроме того, алгоритм удобен для СБИС-реализации. Далее, на базе этого алгоритма преложены алгоритмы выделения контуров на полутоновых изображениях.

\section{2. ОСНОВНЫЕ ПОНЯТИЯ}

2D функции $\mathrm{A}_{\mathrm{mn}}$ могут быть пред-ставлены следующим образом ([3]):

$$
\begin{gathered}
A_{m n}=A(m, n, x, y)=f(m, x) \otimes f(n, y)=f(n, y) \otimes \\
f(m, x),
\end{gathered}
$$

где $\mathrm{m}, \mathrm{n}=0, \ldots, \mathrm{N}-1, \mathrm{f}(\mathrm{m}, \mathrm{x})$ и $\mathrm{f}(\mathrm{n}, \mathrm{y})-$ строчный и столбцовый векторы значений соответствующих функций $\mathrm{f}_{\mathrm{m}}$ и $\mathrm{f}_{\mathrm{n}}$ ортогонального преобразования $\mathrm{A}_{\mathrm{N}} \otimes-$ символ Кронекерова умножения, $\mathrm{N}$ - размерность преобразования, $\mathrm{m}, \mathrm{n}$ - номера функций.

Пусть матрица значений ДЭФ для $\mathrm{N}=4$ имеет вид [1]:

$\mathrm{A}_{4}=\left[\begin{array}{cccc}1 & 1 & 1 & 1 \\ 1 & w_{1} & w_{2} & w_{3} \\ 1 & w_{2} & w_{4} & w_{6} \\ 1 & w_{3} & w_{6} & w_{9}\end{array}\right]=$

$\begin{array}{r}\operatorname{def}_{0} \\ \operatorname{def}_{2} \\ \operatorname{def}_{3}\end{array}\left[\begin{array}{llll}w_{0} & w_{0} & w_{0} & w_{0} \\ w_{0} & w_{1} & w_{2} & w_{3} \\ w_{0} & w_{2} & w_{0} & w_{2} \\ w_{0} & w_{3} & w_{2} & w_{1}\end{array}\right]$,


где $\mathrm{w}=\exp (-\mathrm{j} 2 \mathrm{p} / \mathrm{N})$. Тогда, например, имеем следующую 2D функцию:

$$
A_{20}=\operatorname{def}(2,0, x, y)=\operatorname{def}(0, y) \otimes \operatorname{def}(2, x)=
$$$$
=\left[\begin{array}{l}
w_{0} \\
w_{0} \\
w_{0} \\
w_{0}
\end{array}\right] \otimes\left[\begin{array}{llll}
w_{0} & w_{2} & w_{0} & w_{2}
\end{array}\right]=
$$$$
\left[\begin{array}{llll}
w_{0} & w_{2} & w_{0} & w_{2} \\
w_{0} & w_{2} & w_{0} & w_{2} \\
w_{0} & w_{2} & w_{0} & w_{2} \\
w_{0} & w_{2} & w_{0} & w_{2}
\end{array}\right]
$$

(аналогичным образом можно построить любую другую двумерную функцию $\mathrm{A}_{\mathrm{mn}}$ ).

Как следует из (1), 2D функции $\operatorname{def}(\mathrm{m}, \mathrm{n}, \mathrm{x}, \mathrm{y})$ имеют как одинаковые столбцы, так и столбцы, отличающиеся на соответствующие значения w. Функции, расположенные в столбцах, имеют как одинаковые строки, так и строки, отличающиеся на соответствующие значения w. Эти свойства 2D функций используются для организации параллельных вычислений одинаковые компоненты можно вычислять только один раз и при необходимости использовать ранее полученные результаты.

\section{3. АЛГОРИТМ ПРЕОБРАЗОВАНИЯ}

Пусть дана матрица сигналов отсчета $\mathrm{S}_{\mathrm{N}}=$ $\left[\mathrm{s}_{\mathrm{ij}}\right], \mathrm{i}, \mathrm{j}=0, \ldots, \mathrm{N}-1$, и $(\mathrm{NrN})-$ массив состоит из матриц значений $2 \mathrm{D}$ функций $\mathrm{A}_{\mathrm{mn}}=\left[a_{i j}^{m n}\right], \mathrm{m}, \mathrm{n}$, $\mathrm{i}, \mathrm{j}=0, \ldots, \mathrm{N}-1$. Требуется определить все элементы $\mathrm{f}_{\mathrm{mn}}$ матрицы коэффициентов $\mathrm{F}$. Коэффициенты $\mathrm{f}_{\mathrm{mn}}$ определяются следующим образом $[3,4]$ :

$$
\mathrm{f}_{\mathrm{mn}}=\sum_{i=0}^{N-1} \sum_{j=0}^{N-1} s_{i j} a_{i j}^{m n}
$$

Заметим, что

$$
\begin{aligned}
\mathrm{g}_{n}^{i} & =\sum_{k=0}^{N-1} s_{i k} a_{i j}^{n n}, \\
h_{n}^{j} & =\sum_{k=0}^{N-1} s_{k j} a_{k j}^{n n}, \mathrm{n}=0, \ldots, \mathrm{N}-1 .
\end{aligned}
$$

Легко увидеть, что $h_{n}^{j}$ и $g_{n}^{i}$ имеют одинаковые слагаемые и могут быть вычислены параллельно. Мы используем следующий алгоритм для вычисления $h_{n}^{j}$ и $g_{n}^{i}$.

0 . Начало:

$\mathrm{j}=0$;

$d_{0}^{i}=0$ для $\mathrm{i}=0, \ldots, \mathrm{N}-1$.

1. $\mathrm{C}_{\mathrm{o}}=0$.

2. $U_{k-1 j}=S_{k-1 j} a_{k-1 j}^{n n}$,

$\mathrm{C}_{\mathrm{k}}=\mathrm{C}_{\mathrm{k}-1}+U_{k-1 j}$

$$
d_{j+1}^{k-1}=d_{j}^{k-1}+U_{k-1 j}
$$

для $\mathrm{k}=1,2, \ldots, . \mathrm{N}$.

$h_{n}^{j}=C_{N}$.

$g_{n}^{i}=d_{N}^{i}$.

3. $\mathrm{j}=\mathrm{j}+1$; если $\mathrm{j}<\mathrm{N}$, перейти к 1 .

\section{4. Конец.}

Получив и, мы можем вычислить диагональные элементы $\mathrm{f}_{\mathrm{nn}}$ матрицы $\mathrm{F}$ :

$$
f_{n n}=\sum_{j=0}^{N-1} h_{n}^{j} \text { or } f_{n n}=\sum_{i=0}^{N-1} g_{n}^{i} .
$$

Заметим, что соответствующие слагаемые всех коэффициентов $\mathrm{f}_{\mathrm{mn}}$ для $\mathrm{m}>\mathrm{n}$ получаются аналогично вычислению следующим образом:

$$
f_{m n}=\sum_{j=0}^{N-1} h_{n}^{j} a_{m-n j},
$$

где - элементы матрицы $\mathrm{A}_{\mathrm{mn}}$, которые составляют двумерный базис.

Вычислив, мы можем вычислить все элементы $f_{m}$ матрицы $F$, которые расположены в строке $\mathrm{n}$ справа от диагонального элемента $\mathrm{f}_{\mathrm{nn}}$. Для этого мы используем формулу (9). Аналогично, вычислив, мы можем вычислить все коэффициенты $f_{n m}$ для $m>n$, которые расположены в столбце $\mathrm{n}$ ниже $\mathrm{f}_{\mathrm{nn}}$ :

$$
f_{n m}=\sum_{i=0}^{N-1} g_{n}^{i} a_{m-n i}
$$

Если $\mathrm{n}=0$, то коэффициенты $\mathrm{f}_{\mathrm{m} 0}$ и $\mathrm{f}_{0 \mathrm{~m}}$ есть элементы соответственно 0-й строки и 0-го столбца матрицы F. Вычислив их по формулам (9) and (10), размерность задачи $\mathrm{N}$ уменьшается 
на 1. Далее, при для $\mathrm{n}=1,2, \ldots, \mathrm{N}-1$ находятся другие элементы матрицы F. Таким образом, алгоритм вычисления коэффициентов состоит из $\mathrm{N}$ итераций. Следующие операции выполняются на i-й ит ерации, $\mathrm{i}=0, \ldots, \mathrm{N}-1$ :

1. Вычислить и по формулам (3) - (7).

2. Построить $\mathrm{f}_{\mathrm{nn}}$; если $\mathrm{n}$ №N-1,

то построить $\mathrm{f}_{\mathrm{nm}}$ и $\mathrm{f}_{\mathrm{nm}}$ для всех $\mathrm{n}<\mathrm{mJ} \mathrm{N}-1$.

Следует заметить, что на каждой итерации вычисляются 2(N-n)-1 элементов матрицы F.

Описанный выше алгоритм реализует $2 \mathrm{D}$ функции преобразо-вания Фурье, но он может быть использован для вычисления ДЭФ и для вычисления 2D функций других ортогональных базисов. Для этого сле дует изменить формулы (9) и (10) в соответствии со спецификой базиса.

Заметим, что алгоритм может быть эффективно реализован на СБИС в виде матричной систолической структуры.

Далее рассмотрим использование этого алгоритма для выделения контуров на полутоновых изображениях.

\section{4. ВЫДЕЛЕНИЕ КОНТУРОВ НА ПОЛУТОНОВЫХ ИЗОБРАЖЕНИЯХ}

Определение контуров занимает центральное место при обработке информации в системах технического зрения, представляя собой начальный и решающий этап в распознавании и идент ификации объектов. В частности, выделение контуров применяется при решении задачи идентификации объектов по их форме, при наложении и совмещении разных слоев одного объекта, а также при сегментации изображений.

Все методы и алгоритмы нахождения контуров объектов можно разделить на две категории [4-6]:

1) Методы, основанные на выделении областей, включают такие алгоритмы как "расширение области" за счет объединения пикселей и алгоритм "разбиения и объединения областей”. Эти алгоритмы гарантируют получение замкнутых контурных линий, однако, они чувствительны к шумам и контрастности изображения.

2) Методы, основанные на выделении краевых точек на изображении, включают в себя градиентные методы, операторы Собеля, Робертса, Лапласа, детектор контуров Канни, детектор разрывов Ли и др. Эти методы, как правило, не чувствительны к шумам и контрастности изображения, но требуют применения алгоритма объединения краевых точек на следующем этапе, что также не всегда гарантирует выделение замкнутых контуров.

Эффективность методов трудно оценить, изза сложности определения наилучших параметров каждого из них и отсутствия универсального критерия эффективности $[4,7]$. Тем не менее, различные операторы могут быть эффективными для различных типов изображений. Ниже мы приведем использование операторов Уолша и Хаара для выделения контуров на полутоновых изображениях. Эффективность этих операторов определяется существованием быстрого алгоритма его вычисления - в заданное время контура выделяются с достаточным качеством.

\section{1. Операторы Уолша для выделения контуров}

2D функции Уолша могут применяться непосредственно для выделения контуров. На pис.1 показаны четыре такие функции [8].

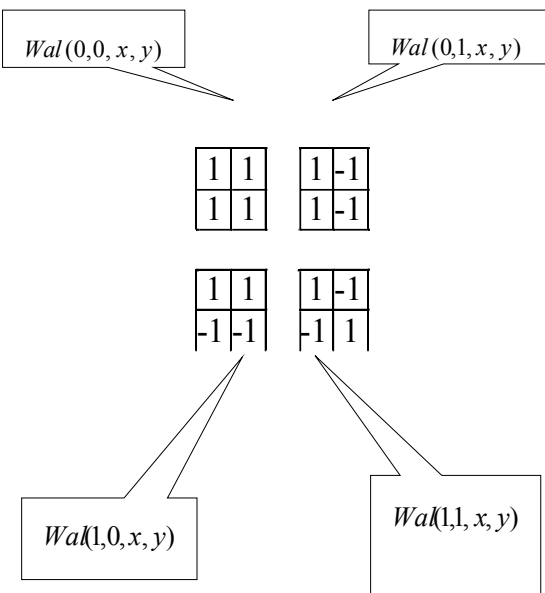

Рис. 1. 2D функции Уолша

Значения коэффициентов $C_{0 I}, C_{10}, C_{11}$ соответствуют функциям, , и вычисляются, когда выполняется прямое преобразование, а коэффициенту $C_{00}$ присваивается нулевое значение. При выполнении обратного преобразования вычисляется только значение коэффициента $C_{00}$. При этом коэффициенты $C_{01}$, $C_{10}, C_{11}$ могут принимать значения по модулю или их истинные значения в зависимости от распределения интенсивностей на изображении, полученном после преобразования. Если используются истинные значения, то вычисленный коэффициент $C_{00}$ должен браться по модулю.

При использовании этого алгоритма вся область изображения сканируется окном 2ґ2 с шагом 1. Значение коэффициента $C_{00}$ 
присваивается пикселю, который соответствует этому коэффициенту в окне. После применения этого алгоритма получается также полутоновое изображение. Граничные точки выбираются посредством порогового разделения по величине значения коэффициента $C_{00}$.

Для выделения контуров обычно используются два матричных оператора размерностью $3 \times 3$ пикселя [4,5], которые могут быть получены из 2D функций Уолша. Для этого в двумерные дискретные функции вставляется строка и столбец с нулевыми элементами. На рис.2 представлены полученные операторы.

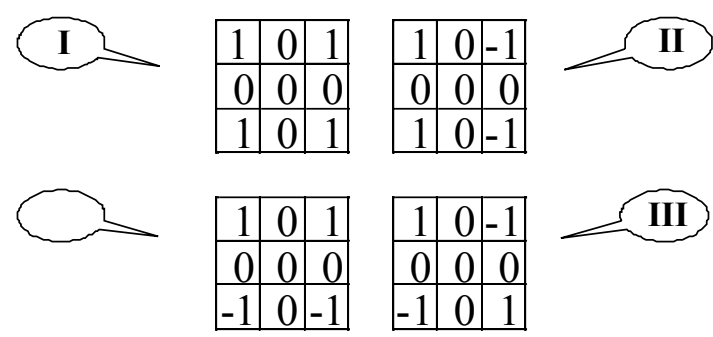

Рис. 2. Операторы Уолиа

Операторы Уолша используются так же, как и известные операторы. Контрастирование перепадов оператором $S(m, n)$ предполагает нелинейный подсчет значений пикселей в окне размером $3 \times 3$ для преобразования значения пикселя в точке $(m, n)$ (см. рис.3).

\begin{tabular}{|c|c|c|}
\hline$f_{m-1, n-1}$ & $f_{m-1, n}$ & $f_{m-1, n+1}$ \\
\hline$f_{m, n-1}$ & $f_{m, n}$ & $f_{m, n+1}$ \\
\hline$f_{m+1, n-1}$ & $f_{m+1, n}$ & $f_{m+1, n+1}$ \\
\hline
\end{tabular}

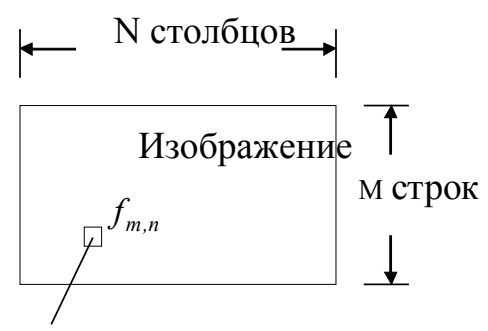

Значение интенсивности пиксела в точке $(\mathrm{m}, \mathrm{n})$

\section{Рис. 3. Обозначения элементов для оператора выделения перепадов}

Его значение определяется как

$$
S(m, n)=\sqrt{d_{x(m, n)}^{2}+d_{y_{(m, n)}}^{2}}
$$

или для снижения сложности вычислений как

$$
S(m, n)=\left|d_{x(m, n)}\right|+\left|d_{y_{(m, n)}}\right|,
$$

где

$$
\begin{aligned}
d_{y_{(m, n)}}= & \left(f_{m+1, n-1}+2 f_{m+1, n}+f_{m+1, n+1}\right) \\
& -\left(f_{m-1, n-1}+2 f_{m-1, n}+f_{m-1, n+1}\right), \\
d_{x(m, n)}= & \left(f_{m-1, n+1}+2 f_{m, n+1}+f_{m+1, n+1}\right) \\
& -\left(f_{m-1, n-1}+2 f_{m, n-1}+f_{m+1, n-1}\right),
\end{aligned}
$$

Оператор перемещается по всей области изображения с шагом 1.

При выделении контуров с применением операторов Уолша возможны любые сочетания операторов, представленных на рис. 2. Наиболее эффективным оказалось применение операторов II и III. При этом значение пикселя в точке $(m, n)$ вычисляется следующим образом:

$$
S(m, n)=\sqrt{X^{2}+Y^{2}},
$$

или, с целью сокращения вычислений:

$$
\begin{gathered}
\quad S(m, n)=|X|+|Y|, \\
\text { где } \\
X=\left(f_{m-1, n-1}+f_{m+1, n-1}\right)-\left(f_{m-1, n+1}+f_{m+1, n+1}\right), \\
Y=\left(f_{m-1, n-1}+f_{m-1, n+1}\right)-\left(f_{m+1, n+1}+f_{m+1, n-1}\right)
\end{gathered}
$$

Для нахождения значений $X$ и $Y$, соответствующих операторам II и III Уолша, можно использовать быстрый алгоритм вычислений.

При использовании этих операторов вся область изображения сканируется окном $3 \times 3$ с шагом 1. После применения оператора получается полутоновое изображение, на котором граничные точки определяются порогом, который задается значением $S(m, n)$. Выбор порога очень важен для окончательного результата [9].

Так же в качестве операторов выделения контуров можно применять двумерные функции Уолша классическим способом. Для этого при выполнении прямого преобразования вычис- 
ляются значения коэффициентов или двумерные функции $\operatorname{wal}(0,1, x, y), \operatorname{wal}(1,0, x, y)$, $\operatorname{wal}(1,1, x, y)$. Коэффициенту $\operatorname{wal}(0,0, x, y)$ присваивается значение нуля. А при выполнении обратного преобразования вычисляется лишь значение коэффициента . Для вычисления $\operatorname{wal}(0,0, x, y)$ коэффициенты $\operatorname{wal}(0,1, x, y), \quad w a l(1,0, x, y), \quad w a l(1,1, x, y)$ могут браться либо по модулю, либо их истинные значения. Отличия будут заключаться в диапазоне распределения яркостей изображения, получаемого после преобразования и соответственно в значении порога, выбираемого для бинаризации изображения. Если использовать истинные значения, то коэффициент wal $(0,0, x, y)$ тогда необходимо брать по модулю. Причем его истинное значение отражает направление перехода в месте перепада: с более светлого на темное или наоборот. При применении такого оператора вся область изображения сканируется окном размером $2 \times 2$ пикселя с шагом равным 1 . Значение коэффициента $\operatorname{wal}(0,0, x, y)$ присваивается пикселю, соответствующему этому коэффициенту в окне оператора.

После выполнения оператора получается полутоновое изображение. Для его бинаризации применяем пороговую обработку.

\section{2. Операторы Хаара для выделения контуров}

Алгоритм выделения контуров на полутоновом изображении с применением 2D функций Хаара состоит из нескольких этапов.

На первом этапе выполняется видоизменение гистограммы распределения яркостей исходного изображения. Это производится посредством деления значения яркости каждого пикселя изображения на делитель $D=2^{n}$. На практике деление на степень двойки выполняется посредством сдвига регистра, содержащего значение яркости пикселя на 1 битовый разряд вправо. В результате такого преобразования диапазон распределения значений яркости сжимается.

Экспериментально определено, что при делении значения яркости каждого пикселя изображения на не происходит потерь контуров изображения и значительно снижается зашумленность изображения ошибочными краевыми точками. Ошибочные краевые точки возникают вследствие того, что при такой малой размерности оператора $2 \times 2$, он является очень чувствительным к незначительным изменениям яркости.

На втором этапе алгоритма выполняется двумерное прямое и обратное преобразование в ортогональном базисе Хаара. Вся область изображения сканируется окном размером $2 \times 2$ $(4 \times 4)$ пикселя, с шагом сканирования, равным размеру окна. К каждому фрагменту изображения применяется прямое и обратное преобразование Хаара в двумерном базисе [8]:

$$
\begin{aligned}
& f_{m n}=\sum_{i=0}^{N-1} \sum_{j=0}^{N-1} s_{i j} \times h a a p_{j}^{m n}, \quad m, n=\overline{0 \div N-1}, \\
& \text { где } s_{i j} \text { и } \operatorname{haar}_{i j}^{m n}-\text { элементы матриц }
\end{aligned}
$$
соответственно значений сигнала и двумерной функции $\operatorname{haar}(m, n, x, y)$.

Таким образом, получается, что если окно оператора попадает на фрагмент изображения с постоянным значением яркости, то все коэффициенты, кроме первого, после прямого преобразования Хаара равны нулю. Первый коэффициент обнуляется и соответственно после обратного преобразования данный фрагмент изображения заполняется черным цветом. Иначе, если окно оператора попадает на фрагмент изображения, на котором значения яркости изменяются, то коэффициенты прямого преобразования Хаара не будут равны нулю, и соответственно после выполнения обратного преобразования на данном фрагменте изображения появляются белые точки, соответствующие точкам контура. Поскольку на дискретных изображениях нет резких порогов изменения яркости, то не происходит потерь контуров, связанных с попаданием окна оператора точно на границу объекта на изображении.

Далее на третьем этапе с целью устранения шумовых точек применяется низкочастотная фильтрация с помощью шумоподавляющей маски:

$$
H=\frac{1}{9}\left[\begin{array}{lll}
1 & 1 & 1 \\
1 & 1 & 1 \\
1 & 1 & 1
\end{array}\right] .
$$

Вся область изображения сканируется данной маской размерности $3 \times 3$ с шагом равным 1, в результате чего получается изображение с выделенными контурными точками. 
Получаемые при таком преобразовании контура не являются замкнутыми, поэтому необходимо выполнение операции объединения краевых точек в замкнутые контура.

\section{ЗАКЛЮЧЕНИЕ}

Использование двумерных базисов обеспечивает непосредственное выполнение соответствующих двумерных преобразований, a предложенный алгоритм быстрого вычисления существенно сокращает вычислительные затраты. На основании этого алгоритма можно синтезировать специализированные аппаратные средства параллельной обработки.

Из множества ортогональных преобразований рассмотрены дискрет-ные функции Уолша и Хаара. Это связано в первую очередь с тем, что базисные функции указанных преобразований принимают значения $+1,-1$ и $+1,-1,0$ соответственно и естественным образом отображаются на элементы цифровой вычислительной техники и дают дополнительные возможности для ускорения вычислений.

Предложены быстрые алгоритмы выделения контуров на основе функций Уолша и Хаара, которые по сравнению с градиентными алгоритмами дают более узкую контурную линию, т.к. обработка изображения производится окном с меньшим размером и обладают высоким быстродействием.

Кроме того, при использовании функций Уолша нет необходимости в пороговой обработке и соответственно выборе величины порога, что является одним из ключевых вопросов при определении контуров градиентными операторами, а функции Хаара позволяют выделять контура на слабоконтрастных изображениях.

\section{ЛИТЕРАТУРА}

[1] K.S. Fu. VLSI for pattern recognition and image processing. Spinger-Verlag, Berlin Heidelberg, New York, Tokyo (1984).

[2] Хармут Х.Ф. Теория секвентного анализа. Основы и применения. - М.: Мир, 1980. - 574 с.

[3] Machnev A.G., Sadykhov R.H. Algorithm and structure of a unit for computing a two-dimensional Vilenkin-Chrestenson transform in signal processing applications.- Proceedings of the Latvian signal processing international conference, vol. 1, $p$ 316-320 (1990).

[4] Прэтт У. Цифровая обработка изображений: Пер. с англ. - М.: Мир, 1982. Kн. 2. -480 c.

[5] K.S. Fu, R.C. Gonzalez, C.S.G. Lee. Robotics: Control, sensing, vision and intelligence. New York etc.: McGraw-Hill, 1987. - XIII.

[6] P.C. Yuen, G.C. Feng, J.P. Zhou. A contour detection method: Initialization and contour model.// Pattern Recognition Letters, Vol. 20, (1999) pp.141-148.

[7] A. Koschan, A Comparative Study on Color Edge Detection. // Reprintfrom Proc. $2^{\text {nd }}$ Asian Conference on Computer Vision ACCV'95, Singapore, 5-8 December 1995, Vol. III, pp. 574-578.

[8] Садыхов Р.Х., Мачнев А.Г. Систолические процессоры иифровой обработки изображений в двумерных базисах. - Минск: Ин-т техн. Кибернетики АН Беларуси, 1996. - 82c.

[9] P.L.Rosin, Edges: saliency measu-res and automatic thresholding. // Mach. Vision Appl. 9, pp. 139-159 (1997).

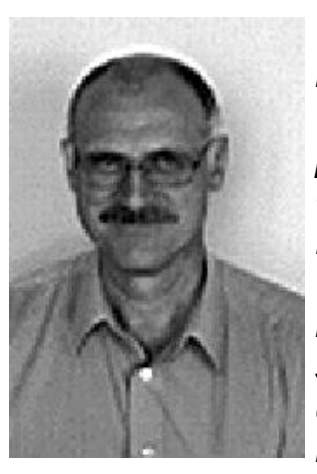

Дудкин Александр Арсентьевич

Ведущий научный comрудник Институmа технической кибернетики НАН Беларуси.

Кандидат технических наук с 19872., имеет ученое звание старшего научного сотрудника по спец. «Системы автоматизированного проектирования».

Область научных интересов: методы и алгоритмы синтеза цифровых интегральных схем, системы автоматизированного проектирования больших интегральных схем, цифровая обработка сигналов и изображений, распознавание образов, архитектуры и модели систем компьютерного зрения и высокопроизводительной обработки информации.

В Инстumyme paбоmaem c 1981 г.: в лаборатории системного программирования и логического проектирования, с 1989 г. - в лаборатории проблем построения САПР СБИС, с 1994 2. - в лаборатории Идентификации систем.

Автор 50 научных публикаций.

Член белорусского отделения международного общества нейронных сетей, член IEE u Белорусской ассоциации по распознаванию изображений (БААРИ).

doudkin@newman.bas-net.by

http://lsi.bas-net.by/doudkin

Раб. Тел. 375 (17) 2842164 


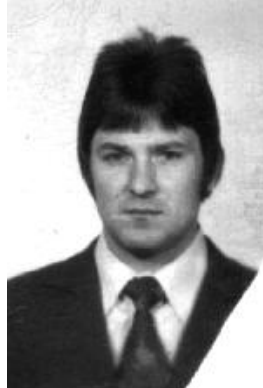

Мачнев Александр Григорьевич

Научный сотрудник лаборатории Идентифрикации систем Института mexнической кибернетики НАН Беларуси.

Кандидат технических наук с 1998 г.

Область научных интересов: цифровая обработка сигналов и изображений, параллельные архитектуры для цифровой обработки сигналов и изображений, алгоритмы и вычислительные структуры двумерных ортогональных преобразований.

Автор 30 научных публикаций и 20 авторских свидетельств.

Селиханович Андрей Михайлович

Младший научный сотрудник лаборатории Идентифрикации систем Института технической кибернетики НАН Беларуси.

Область научных интересов: двумерные ортогональные функции, выделение контуров, обработка изображений, распознавание образов.

В лаборатории работает с 1998 г. после окончания аспирантуры Института. 